\title{
THE SECOND FUNDAMENTAL THEOREM FOR MEROMORPHIC MINIMAL SURFACES ${ }^{1}$
}

\author{
BY E. F. BECKENBACH AND T. A. COOTZ
}

Communicated by M. H. Protter, January 12, 1970

\begin{abstract}
The second fundamental theorem of Nevanlinna concerning meromorphic functions of a complex variable is extended in this note to an analogous result for meromorphic minimal surfaces. A similar extension of the first fundamental theorem involved generalizations of the classical proximity and enumerative functions and also a new visibility function; for the present result, a second enumerative function and a second visibility function are defined. Defect relations are discussed.
\end{abstract}

1. Introduction. The second fundamental theorem of Nevanlinna concerning meromorphic functions of a complex variable $[4$, p. 227] is extended in this note to an analogous result for meromorphic minimal surfaces. A full presentation of the material here briefly outlined will appear elsewhere. Details of the proof of a similar extension of the first fundamental theorem can be found in [1].

Let a surface

$$
S: x_{j}=x_{j}(u, v), \quad j=1,2,3,
$$

be given in isothermal representation, that is, in a representation for which

$$
E(u, v)=G(u, v), \quad F(u, v)=0,
$$

where $E, F$, and $G$ are the coefficients of the first fundamental quadratic form of $S$. Then $S$ is a minimal surface if and only if the coordinate functions (1) are harmonic.

If $S$ is a minimal surface in isothermal representation, then the poles and the finite a-points, where $a=\left(a_{1}, a_{2}, a_{3}\right)$, of $S$ are isolated, as are the infinities and the zeros of the area-deformation function $E[1]$.

A meromorphic minimal surface is a minimal surface, with harmonic coordinate functions (1) satisfying (2), which has no singularities other than poles for $u^{2}+v^{2}<\infty$.

2. The first fundamental theorem. In the stereographic projection of extended Euclidean 3-space onto the hypersphere

AMS Subject Classifications. Primary 5310; Secondary 3068, 3070, 3105.

Key Words and Phrases. Minimal surface, meromorphic function, Nevanlinna theory, defect relation, second fundamental theorem.

1 This research was supported in part by NSF Grant \#GP-13066. 


$$
V: x_{1}^{2}+x_{2}^{2}+x_{8}^{2}+\left(x_{4}-\frac{1}{2}\right)^{2}=\left(\frac{1}{2}\right)^{2}
$$

let $\chi(x, a)$ denote the chordal distance between the images of $\mathbf{x}$ and $\boldsymbol{a}$, so that

$$
\begin{aligned}
\chi(x, \infty) & =\frac{1}{(1+x \cdot x)^{1 / 2}} \\
\chi(x, a) & =\frac{[(x-a) \cdot(x-a)]^{1 / 2}}{(1+x \cdot x)^{1 / 2}(1+a \cdot a)^{1 / 2}}
\end{aligned}
$$

for a finite. In analogy with the Ahlfors-Shimizu spherical version of the complex-variable case, for a meromorphic minimal surface $S$, and for a finite or infinite, we define a hyperspherical proximity function by

$$
m^{\circ}(r, a ; S)=\frac{1}{2 \pi} \int_{u^{2}+v^{2}=r^{2}} \log \frac{1}{\chi(\mathrm{x}, \boldsymbol{a})} d \theta .
$$

Again in analogy with the classical theory, for a finite or infinite we let $n(t, a ; S)$ denote the sum of the orders of the a-points of $S$ in $u^{2}+v^{2} \leqq t^{2}$ and define an enumerative function for $S$ by

$$
N(\boldsymbol{r}, \boldsymbol{a} ; S)=\int_{0}^{r} \frac{n(t, \boldsymbol{a} ; S)-n(0, \boldsymbol{a} ; S)}{t} d t+n(0, \boldsymbol{a} ; S) \log r .
$$

In the extended theory, we let

$$
\begin{aligned}
h(t, \infty ; S) & =0 \\
h(t, a ; S) & =\frac{1}{\pi} \iint_{u^{2}+v^{2} s^{2}} \frac{[(x-a) \cdot X]^{2}}{[(x-a) \cdot(x-a)]^{2}} E d u d v
\end{aligned}
$$

for a finite, where $X(u, v)$ denotes the unit normal to $S$, and adjoin to the proximity and enumerative functions a visibility function for $S$, defined for a finite or infinite by

$$
H(\boldsymbol{r}, \boldsymbol{a} ; S)=\int_{0}^{r} \frac{h(t, \boldsymbol{a} ; S)}{t} d t .
$$

The hyperspherical affinity of $S$ to $a$ in $u^{2}+v^{2} \leqq r^{2}$, or the hyperspherical affinity function for $S$, is defined by

(9) $\mathfrak{U}^{\circ}(\boldsymbol{r}, \boldsymbol{a} ; S)=m^{\circ}(\boldsymbol{r}, \boldsymbol{a} ; S)+N(\boldsymbol{r}, \boldsymbol{a} ; S)+H(\boldsymbol{r}, \boldsymbol{a} ; S)+C(\boldsymbol{a} ; S)$,

where the constant $C(a ; S)$ is chosen so that $\lim _{r \rightarrow 0} \mathfrak{X}^{\circ}(r, a ; S)=0$. In particular, the hyperspherical affinity of $S$ to $\infty$ is called the hyperspherical characteristic function for $S$ and is denoted by $T^{\circ}(r ; S)$.

The generalization [1] of the Ahlfors-Shimizu spherical form of the 
first fundamental theorem of Nevanlinna to meromorphic minimal surfaces is the following:

$$
T^{\circ}(r ; S)=\mathfrak{H}^{\circ}(r, \boldsymbol{a} ; S) \text { for all finite } \mathbf{a} .
$$

For any nonconstant meromorphic minimal surface $S$, the function $T^{\circ}(r ; S)$ is positive for $r>0$ and is an increasing, strictly convex function of $\log r$. The function $N(r, a ; S)$ is a nondecreasing, piecewise linear, convex function of $\log r$. The function $H(r, a ; S)$ vanishes identically if $a$ is infinite or if $S$ is a plane surface and a lies in the plane; otherwise, for $r>0, H(r, a ; S)$ is a positive, increasing, strictly convex function of $\log r$.

3. The second fundamental theorem. For an extension of the second fundamental theorem, we introduce a second enumerative function $N_{1}(r ; S)$ and a second visibility function $H_{1}(r ; S)$.

First, following the classical theory, we let

$$
n_{1}(t ; S)=n\left(t, 0 ; S_{u}\right)-n\left(t, \infty ; S_{u}\right)+2 n(t, \infty ; S),
$$

where $0=(0,0,0)$ and the surface $S_{u}$ is defined by

$$
S_{u}: x_{j}=\partial x_{j}(u, v) / \partial u, \quad j=1,2,3 .
$$

The function $n_{1}(t ; S)$ registers the multiple points of $S$ in the disc $u^{2}+v^{2} \leqq t^{2}$, in such a manner that a finite or infinite $k$-fold point of $S$ contributes $k-1$ to $n_{1}(t ; S)$. We then define the enumerative function $N_{1}(r ; S)$ by

Next we let

$$
\begin{aligned}
N_{1}(r ; S) & =\int_{0}^{r} \frac{n_{1}(t ; S)-n_{1}(0 ; S)}{t} d t+n_{1}(0 ; S) \log r \\
& =N\left(r, 0 ; S_{u}\right)-N\left(r, \infty ; S_{u}\right)+2 N(r, \infty ; S) .
\end{aligned}
$$

$$
h_{1}(t ; S)=\frac{1}{2 \pi} \iint_{u^{2}+v^{2} \leq t^{2}}(-E K) d u d v
$$

where $K(u, v)$ denotes the Gaussian curvature of $S$. We note in passing that for a minimal surface $S$ we have $K \leqq 0$ so that $-E K \geqq 0$, and in fact that $-K$ is the area-deformation ratio for the map of $S$ onto its spherical (Gaussian) representation [3, p. 253]; thus $2 \pi h_{1}(t ; S)$ expresses the area of the map of $u^{2}+v^{2} \leqq t^{2}$ on the Gaussian sphere for $S$. We now define the visibility function $H_{1}(r ; S)$ by

$$
\begin{aligned}
H_{1}(r ; S) & =\int_{0}^{r} \frac{h_{1}(t ; S)}{t} d t \\
& =\frac{1}{2 \pi} \int_{0}^{r}\left[\iint_{u^{2}+v^{2} s t^{2}}(-E K) d u d v\right] \frac{d t}{t} .
\end{aligned}
$$


A generalization of the second fundamental theorem of Nevanlinna to meromorphic minimal surfaces can now be stated as follows:

Let $S$ be a nonconstant meromorphic minimal surface and let $a_{1}, a_{2}$, $\cdots, a_{q}$ be $q$ points, $q>2$. Let $k$ be a given real number, $k \geqq 0$. Then

$$
\begin{aligned}
\sum_{j=1}^{q} m^{\circ}\left(r, a_{j} ; S\right) \leqq & 2 T^{\circ}(r ; S)-N_{1}(r ; S)-H_{1}(r ; S) \\
& +O[\log r]+O\left[\log T^{\circ}(r ; S)\right]
\end{aligned}
$$

for $r$ outside an open set $\Delta_{k}$ such that $\int_{\Delta_{k}} r^{k} d r<\infty$.

4. Indication of proof. On the hypersphere $V$ given by (3), the mass distribution

$$
\rho(a)=C\left[\prod_{j=1}^{q} \frac{2}{\chi\left(a, a_{j}\right)}\right]^{3}\left[\log \prod_{j=1}^{q} \frac{2}{\chi\left(a, a_{j}\right)}\right]^{-\alpha}, \quad \alpha>1,
$$

is positive for $C>0$ and is continuous except at the points $a_{j}$; further, with $C$ suitably chosen, $\rho$ is of total mass 1 .

Multiplying both sides of (10) by $\rho(a)$ and integrating over $V$, by (9) we obtain

$$
T^{\circ}(r ; S)=m_{\rho}^{\circ}(r ; S)+N_{\rho}(r ; S)+H_{\rho}(r ; S)+C_{\rho}(S),
$$

with

$$
H_{\rho}(r ; S)=\iiint_{V} H(r, \boldsymbol{a} ; S) \rho(\mathbf{a}) d V_{\mathbf{a}}
$$

and analogous expressions for $m_{\rho}^{\circ}(r ; S), N_{\rho}(r ; S)$, and $C_{\rho}(S)$, where $d V_{\mathrm{a}}$ is the element of volume on $V$.

Since $N(r, a ; S)=0$ except on a set of 3-dimensional measure 0 , we have $N_{\rho}(r ; S) \equiv 0$. For $r_{0}$ fixed, $r_{0}>0$, and for $r>r_{0}, m_{\rho}^{\circ}(r ; S)$ and $T^{\circ}\left(r_{0} ; S\right)$ are positive, and accordingly (18) yields

$$
H_{\rho}(r ; S)-H_{\rho}\left(r_{0} ; S\right)<T^{\circ}(r ; S)+m_{\rho}^{\circ}\left(r_{0} ; S\right) .
$$

Now by (7), (8), and (19), we have

$$
H_{\rho}(r ; S)-H_{\rho}\left(r_{0} ; S\right)=\int_{r_{0}}^{r}\left[\int_{0}^{t} \lambda(\tau ; S) \tau d \tau\right] \frac{d t}{t},
$$

where

$$
\lambda(\tau ; S)=\frac{1}{2 \pi} \int_{u^{2}+v^{2}=\tau^{2}} E \sigma d \theta
$$


with

$$
\sigma(u, v)=\iiint_{V} \frac{2[(\mathbf{x}-\mathbf{a}) \cdot \boldsymbol{X}]^{2}}{[(\mathbf{x}-\mathbf{a}) \cdot(\mathrm{x}-\mathbf{a})]^{2}} \rho(\mathbf{a}) d V_{\mathbf{a} .}
$$

From (22) and the inequality between the geometric mean and the arithmetic mean, we obtain

$$
\log \lambda(r ; S) \geqq \frac{1}{2 \pi} \int_{u^{2}+v^{2}=r^{2}} \log E d \theta+\frac{1}{2 \pi} \int_{u^{2}+v^{2}=r^{2}} \log \sigma d \theta .
$$

We shall now determine values or estimates for the three terms in (24).

The form of the relations (20) and (21) is exactly the same as that of relations in the Nevanlinna theory [4, p. 224], so we may use methods from that theory to show $[4, \mathrm{pp} .226,227]$ that

$$
\log \lambda(r ; S)=O[\log r]+O\left[\log T^{\circ}(r ; S)\right]
$$

for $r$ outside an open set $\Delta_{k}$ such that $\int_{\Delta_{k}} r^{k} d r<\infty$.

The first integral in the right-hand member of (24) can be evaluated by an application of Green's theorem. This gives

$$
\begin{aligned}
\frac{1}{2 \pi} \int_{u^{2}+v^{2}=r^{2}} \log E d \theta= & 2 N\left(r, 0 ; S_{u}\right)-2 N\left(r, \infty ; S_{u}\right) \\
& +\frac{1}{2 \pi} \int_{0}^{r}\left(\iint_{u^{2}+v^{2} \leqq t^{2}} \Delta \log E d u d v\right) \frac{d t}{t} \\
& + \text { const. }
\end{aligned}
$$

Since $\Delta \log E=-2 E K[2]$, by (13), (15), and (26) we therefore have

$$
\begin{aligned}
\frac{1}{2 \pi} \int_{u^{2}+v^{2}=r^{2}} \log E d \theta= & 2 N_{1}(r ; S)-4 N(r, \infty ; S) \\
& +2 H_{1}(r ; S)+\text { const. }
\end{aligned}
$$

Substituting from (17) into (23), integrating over $V$ except for small spherical regions about the $a_{j}$, and taking the limit as the radii of the spheres approach 0 , we find that

(28) $\sigma(u, v)=(1+x \cdot x)^{-2}\left[\prod_{j=1}^{q} \chi\left(x, a_{j}\right)\right]^{-2}\left[\log \prod_{j=1}^{q} \frac{2}{\chi\left(x, a_{j}\right)}\right]^{-\alpha} B(u, v)$,

where $B(u, v)$ is bounded. Thus (cf. [4, p. 228]), by (5), the second integral in the right-hand member of (24) satisfies 


$$
\begin{aligned}
\frac{1}{2 \pi} \int_{0}^{2 \pi} \log \sigma d \theta= & -4 m^{\circ}(r, \infty ; S)+2 \sum_{j=1}^{q} m^{\circ}\left(\boldsymbol{r}, \boldsymbol{a}_{j} ; S\right) \\
& +O(1)+O\left[\log T^{\circ}(\boldsymbol{r} ; S)\right] .
\end{aligned}
$$

Now (16) follows from (24), (25), (27), and (29).

5. Defect relations. In conformity with the classical theory, we define, among other numbers, the following:

$$
\begin{aligned}
\delta(\boldsymbol{a} ; S) & =\liminf _{r \rightarrow \infty}\left(m^{\circ}(r, \boldsymbol{a} ; S) / T^{\circ}(r ; S)\right), \\
\Phi(S) & =\liminf _{r \rightarrow \infty}\left(N_{\mathbf{1}}(r ; S) / T^{\circ}(r ; S)\right), \\
\Psi(S) & =\liminf _{r \rightarrow \infty}\left(H_{1}(r ; S) / T^{\circ}(r ; S)\right) .
\end{aligned}
$$

The numbers (30), (31), and (32) are nonnegative, and they satisfy

$$
\begin{gathered}
\delta(\boldsymbol{a} ; S) \leqq 1, \quad \Phi(S) \leqq 2, \quad \Psi(S) \leqq 2, \\
\sum_{\mathbf{a}} \delta(\boldsymbol{a} ; S)+\Phi(S)+\Psi(S) \leqq 2 .
\end{gathered}
$$

These and other related inequalities suggest questions as to the existence of nonplane meromorphic minimal surfaces $S$ for which $\delta(a ; S), \Phi(S)$, and $\Psi(S)$ are related in specified ways. For example, Richard $E$. Tafel has shown, in his as yet unpublished doctoral dissertation, that for any given $\delta_{0}, 0<\delta_{0}<2$, there is a nonplane meromorphic minimal surface $S$ for which $\sum_{a} \delta(a ; S)=\delta_{0}$.

\section{REFERENCES}

1. E. F. Beckenbach and G. A. Hutchison, Meromorphic minimal surfaces, Pacific J. Math. 28 (1969), 17-47. MR 38 \#6473.

2. E. F. Beckenbach and T. Rad6, Subharmonic functions and surfaces of negative curvature, Trans. Amer. Math. Soc. 35 (1933), 662-674.

3. L. P. Eisenhart, An introduction to differential geometry, Princeton Math. Series, vol. 3, Princeton Univ. Press, Princeton, N. J., 1940. MR 2, 154.

4. E. Hille, Analytic function theory. Vol. II, Introductions to Higher Math., Ginn and Co., Boston, Mass., 1962. MR $34 \# 1490$.

University of California, los Angeles, California 90024

McDonnell-Douglas Astronautics Company, Huntington Beach, CaliFORNIA 92647 